\title{
HUMOR, GÉNERO Y RELACIÓN SOCIAL. EL HUMOR COMO ESTRATEGIA INTERACCIONAL
}

\author{
Magdalena Romera \\ Universidad Pública de Navarra-Universitat de les Illes Balears
}

Recibido: 15/09/2014

Aceptado: 24/11/2014

\section{Resumen}

Este trabajo explora el uso de las expresiones lingüísticas de humor como herramienta interaccional en la conversación, en relación al género de los participantes y la relación social que los une. Mientras que hace algún tiempo se aludía a la falta de sentido del humor por parte de las mujeres, hoy está claro que las mujeres, al igual que los hombres, usan el humor como estrategia fundamental en la interacción. Desde este punto de vista interaccional, se ha aludido además a la importancia de factores sociales y contextuales en la producción e interpretación del humor. En este trabajo nos centramos en la relación social que mantienen los hablantes como elemento que condiciona su conducta discursiva y que por lo tanto puede afectar al uso de las expresiones de humor. A partir de los datos de conversaciones semidirigidas, mantenidas por dos parejas de mujeres, dos parejas de hombres, una pareja mixta y dos grupos de hombres y mujeres, a los que les unen distintas relaciones, analizamos el humor como estrategia discursiva. Teniendo en cuenta que el recurso al humor presenta variaciones individuales, vemos cómo tanto mujeres como hombres lo utilizan fundamentalmente como herramienta para el fortalecimiento de la relación social. Asimismo el grado de fortaleza de los vínculos entre ellos permite un mayor o menor despliegue del modo humorístico de interacción.

Palabras clave: Género; expresión del humor; relación social; interacción discursiva; afiliativo; informacional.

\begin{abstract}
This paper explores the use of linguistic expressions of humor as an interactional tool in conversation, according to the gender of the participants and the social relationship
\end{abstract}


held between them. While early studies argued that women lacked a sense of humor, it is now clear that both women and men use humor as a key strategy in interaction. Besides gender, from an interactional perspective, other social and contextual factors have been claimed to play an important role in the use of humor. In this paper we focus on the social relationship held between speakers as an element that determines their linguistic behavior and which can therefore affect their use of humor in interaction. From semi-structured conversations maintained by two pairs of women, two pairs of men, a couple and two mixed groups of men and women, we analyze the use of humorous expressions as an interactional strategy. Bearing in mind that the use of humor is highly constrained by individual variation, we see how both women and men use humorous expressions primarily as a way of strengthening their social relationships. Also the strength of the bonds holding between them allows them to use a more humorous or a more serious mode of communication.

Keywords: Gender; expression of humor; social relationship; discourse interaction; affiational; informational. 


\section{Introducción}

Este trabajo explora el uso de las expresiones lingüísticas de humor como herramienta interaccional en la conversación, en relación a dos variables concretas, el género de los participantes y la relación social que los une.

Los estudios sobre las expresiones de humor señalan las diferencias en su uso con respecto al género de los hablantes. Mientras que en los primeros estudios se aludía a la falta de sentido del humor en las mujeres, hoy en día se sabe que las diferencias entre ambos no están tanto en su uso, puesto que ambos utilizan el humor como estrategia fundamental en sus interacciones, sino en las distintas funciones con que cada uno las emplea ${ }^{1}$. Dentro de los estudios hispánicos, el grupo GRIALE es quien más detalladamente ha analizado el humor en discursos espontáneos ${ }^{234}$. Sus trabajos han arrojado luz sobre el uso de las expresiones humorísticas, especialmente sobre el recurso a la ironía en la conversación. En esta línea, parece necesario profundizar en aspectos relacionados con las variables sociales que condicionan el uso del humor. En este trabajo queremos ocuparnos de este aspecto, en concreto de comprobar qué uso se hace del humor como estrategia interaccional teniendo en cuenta el género de los hablantes. Además de esto, debido precisamente a la perspectiva interaccional que adoptamos, nos parece necesario contar con otras variables que juegan un papel esencial en la interacción. Aunque la mayoría de los estudios sobre el humor hace referencia a la importancia de los factores sociales y contextuales en

1. KотткоFF, Helga. «Gender and humor: The state of the art». Journal of Pragmatics 38 (2006), pp. 4-25.

2. Ruíz Gurillo, Leonor y Padilla García, Xosé. A. (dirs.). Dime cómo ironizas y te diré quién eres. Una aproximación pragmática a la ironía, Frankfurt, Peter Lang, 2009.

3. Ruíz GuRILlo, Leonor. La lingüística del humor en español, Madrid, Arco/Libros, 2012.

4. Alvarado Ortega, M. Belén. «An Approach to Verbal Humor in Interaction», ProcediaSocial and Behavioral Sciences 95 C, (2013), pp. 594-603. 
la producción e interpretación del mismo ${ }^{5789}$, hasta el momento no se ha explorado la relevancia de la 'relación social' entre los hablantes como factor condicionante. Todo hecho discursivo presenta funciones relacionadas con su propósito comunicativo, así como otras cuyo objetivo es el mantenimiento de la relación social en buenas condiciones ${ }^{10}$. Entendemos por relación social el resultado de los vínculos entre participantes que crean un contexto particular para los interlocutores, en el que el plano colectivo no es la suma de las circunstancias individuales, sino el resultado de la historia conjunta de ambos ${ }^{11}$. De este modo, la relación social crea reglas de conducta particulares, un código relacional propio ${ }^{12}$, que condiciona el uso de las expresiones lingüísticas entre los miembros.

Para explorar estos aspectos nos centramos en el análisis de datos extraídos de cinco conversaciones semidirigidas entre hablantes repartidos en función del género y la relación social que los une. Analizaremos los datos de las conversaciones mantenidas entre dos parejas de mujeres, dos parejas de hombres, una pareja mixta y dos grupos de hombres y mujeres en las que se les pide que discutan sus opiniones acerca de varios temas. Comprobaremos hasta qué punto los factores género y tipo de relación se muestran como condicionantes en el uso de las expresiones de humor.

5. Attardo, Salvatore. Humorous Texts: A Semantic and Pragmatic Analysis, Nueva York, Mouton de Gruyter, 2001.

6. ATtARDO, Salvatore. «A primer for the linguistics of humor.» En Victor Raskin (dir.). The primer of humor research, Berlin, Mouton de Gruyter, 2008.

7. Kerbrat-OreCChiOni, Catherine. «Lhumour au quotidien». En Philip Merlo (dir.) L'humour hispanique, Grimh-LCE-Grimia, Université Lumière Lyon 2, 2004.

8. KotThofF, Helga, 2006, op. cit.

9. Ruíz GURILlO, Leonor, 2012, op. cit.

10. Watzlawick, Paul, Beavin, Janet y Jackson, Don. The Pragmatics of Human Communication. Nueva York, W.W. Norton and Company, 1967.

11. ARUNDAlE, Robert. «Relating», En Miriam Locher y George Sage (dirs.): Interpersonal pragmatics, Berlin, Mouton de Gruyter, (2010), pp. 137-165.

12. Burleson, Brant, MetTS, Sandra y KirCH, Michael W. «Communication in close relationships». En Clyde Hendrick y Susan S. Hendrick (dirs.), Close relationships: A sourcebook, Thousand Oaks, CA, Sage, 2000, pp. 244-258. 


\section{2, Humor y género en la interacción discursiva}

Diversos autores han señalado que el humor es una forma de interacción social ${ }^{13} 14151617$. El modo de interacción humorístico permite interpretar los aspectos de la realidad desde una perspectiva diferente, proporciona un marco compartido en el que tienen cabida las contradicciones, incongruencias y ambigüedades inherentes a toda relación social, y permite por tanto negociar transacciones de otro modo difíciles ${ }^{18}$.

Las funciones principales de las expresiones de humor en la interacción generalizables a diferentes culturas han sido definidas en torno a tres tipos. El destinado a reforzar el vínculo entre interlocutores, también llamado de solidaridad; el relativo a la gestión del poder en la interacción; y el encaminado a servir funciones psicológicas, tales como proteger la imagen propia o gestionar problemas propios o aspectos difíciles 19202122232425 .

La función solidaria parece ser la más frecuente en todos los estudios sobre el humor y está estrechamente ligada al mantenimiento de la interacción y de la propia relación entre los hablantes. En este sentido se ha apelado al principio de la búsqueda del acuerdo ${ }^{26}$. Como señala Pullin $(2010)^{27}$, el consenso

13. RASKIN,Victor. Semantic Mechanisms of Humor. Dordrecht, D. Reidel, 1985.

14. MulKaY, Michael. «On Humour», Cambridge, Polity Press, 1988.

15. ATTARDO, Salvatore, 2001, op. cit.

16. KотTHOFF, Helga, 2001, op. cit.

17. MARTIN, Rod A. The psychology of humor. An integrated approach, Amsterdam, Elsevier, 2007.

18. MARTIN, Rod A., op. cit., p. 115.

19. AlBERTS, Janet. «The use of humor in managing couples' conflict interactions». En Cahn, Dudley D. (dir.). Intimates in conflict. A communicative prespective, Hillsdade, Lawrence Erlbaum, 1990, pp. 105-120.

20. ERVIN-TRIPP, Susan M., LAMPERT, Martin D. «Gender differences in the construction of humorous talk». En Kira Hall, Mary Buchholtz y Birch Moonwomon (dirs.), Locating Power: Proceedings of the Second Berkeley Women and Language Conference. Berkeley, University of California, 1992, pp. 108-117.

21. HAY, Jennifer. «Functions of humor in the conversations of men and women». Journal of Pragmatics 32 (2000), pp. 709-742.

22. KotTHOFF, Helga, 2001, op. cit.

23. HOLMES, Janet. «Sharing a laugh: pragmatic aspects of humor and gender in the workplace». Journal of Pragmatics 38 (2006), pp. 26-50.

24. Ruíz GuRILlo, Leonor, 2012, op. cit.

25. Alvarado Ortega, M. Belén, 2013, op. cit.

26. GRICE, H. Paul. «Logic and conversation». En Peter Cole y Jerry Morgan (dirs.), Syntax and Semantics 3: Speech acts, Nueva York, Academic Press, 1975, pp. 41-58.

27. PUllin, Patricia. "Small talk, rapport, and international communicative competence: Lessons to learn from BELF». Journal of Business Communication, 47 (4) (2010), pp. 455-476.

Feminismo/s 24, diciembre 2014, pp. 41-66 
y la búsqueda de puntos en común refuerzan la solidaridad entre los actantes, revitalizando al mismo tiempo la buena relación entre ellos y suavizando los posibles conflictos anteriores o futuros. Lo interesante es que consenso y solidaridad se retroalimentan. A mayor cercanía y solidaridad establecida y asentada en la relación, mayor es la tolerancia al desacuerdo y la disposición a la resolución del mismo, sin que esto afecte a las partes. El humor es también una estrategia discursiva que permite la gestión del poder en la interacción. Martineau $(1972)^{28}$, Hay $(2000)^{29}$ o Kotthoff $(2006)^{30}$ se refieren a la indexicalización del estatus o la provocación del conflicto en la interacción. En este sentido, el uso de expresiones de humor para la expresión del desacuerdo o incluso para la propia agresión puede estar reservada a aquellos que ostentan el poder en la relación, tanto para aumentar las diferencias como para reducir la asimetría ${ }^{31}{ }^{32}$. El humor también denota poder cuando se emplea para mantener el control de la situación, del comportamiento del otro o de las reglas interaccionales. Finalmente, las funciones psicológicas del humor se centran en el uso del humor para la expresión de aspectos delicados, de los que de otro modo resultaría difícil hablar o que pondrían en peligro la imagen de la persona.

Como vemos, la actividad humorística es una actividad dialógica y sensible a los elementos contextuales. Los factores situacionales, la relación entre los interlocutores, las características de los mismos, edad, sexo, estatus y su disposición y personalidad, harán que el humor obtenga distintas interpretaciones y cumpla funciones diversas.

Entre estos factores se han destacado las diferencias existentes en torno al género como variable transversal que cruza todas las interacciones. Los primeros trabajos en el ámbito anglosajón proponían una incapacidad de la mujer tanto para el uso como para la interpretación del humor ${ }^{33} 34$. Sin embargo, los estudios posteriores han mostrado que esta visión tomaba como

28. MartineAU, William. «A model of the social functions of humor». En Jeffrey Goldstein y Paul McGhee (dirs.), The psychology of humor, Nueva York, Academic Press, 1972, pp. 101-125.

29. HAY, Jennifer, 2000, op. cit.

30. KotTHOFF, Helga, 2006, op. cit.

31. COSER, Rose L. «Laughter among colleagues». Psychiatry 23 (1960), pp. 81-95.

32. KotTHOFF, Helga, 2006, op. cit.

33. LAKOFF, Robin. Language and Woman's Place. NuevaYork, Harper y Row, 1975.

34. Cox, Joe A., Read, L. Raymond y Van Auken, Philip M., «Male-female differences in communicating job-related humor: An exploratory study». Humor 3(1990), pp. 287-295.

35. Goodman, Lizbeth. «Gender and humour». En Frances Bonner et al. (dirs), Imagining women: Cultural representations and gender, Cambridge: Polity, 1992, pp. 296-300. 
referencia un modelo simplista de comunicación, basado únicamente en una perspectiva masculina ${ }^{36}$. Los trabajos más recientes indican que hombres y mujeres utilizan el humor en la interacción, aunque pueden variar las funciones con que cada uno lo emplea.

Una de las características que habitualmente se menciona como típicamente femenina es la orientación más solidaria de la mujer ${ }^{37}$. Los primeros estudios indicaban que las mujeres utilizaban el humor de modo menos agresivo que los hombres ${ }^{38}$. Por el contrario, trabajos más recientes muestran que el humor juega papeles parecidos en ambos, haciendo prevalecer el establecimiento de la solidaridad tanto entre mujeres, como entre hombres, como en grupos mixtos ${ }^{39}{ }^{40}$. A pesar de esto, las estrategias utilizadas pueden ser diferentes, Hay $(2000)^{41}$, Kotthoff $(2000)^{42}$ o Ruíz Gurillo (2012) ${ }^{43}$ coinciden en reportar que las mujeres emplean más alusiones a experiencias propias para crear afinidad con su interlocutor, mientras que los hombres recurren al recuento de experiencias compartidas o alusiones sarcásticas a miembros presentes. Al mismo tiempo la relación social se muestra como un elemento de gran relevancia en las funciones solidarias del humor, prevaleciendo sobre el género de los actantes, de modo que el ataque o la provocación del conflicto mediante el humor solo se permiten cuando la relación está bien establecida ${ }^{44}$. La ironía o la burla aunque sea humorística, siempre pueden resultar amenazadoras y por tanto peligrosas para el mantenimiento de la relación ${ }^{45}{ }^{46}$.

Las diferencias en la indexicalización del poder en la interacción también se muestran asociadas al género. La mayoría de los estudios sugiere que son los hombres los que utilizan el humor para hacer prevalecer su estatus sobre

36. JENKINS, Mercilee. «What's so funny?: Joking among women». En Sue Bremner, Noelle Caskey y Birch Moonwomon (dirs), Proceedings of the first Berkeley Women and Language Conference, Berkeley, CA, Women and Language Group, 1985, pp. 135-151.

37. Deaux, Kay, «Sex and Gender». Annual Review of Psychology 36 (1995), pp. 49-82.

38. Mitchell, J. Clyde. «Network procedures». En Dieter Frick (dir.), The Quality of Urban Life, Berlin, Mouton de Gruyter, 1986, pp. 73-92.

39. HAY, Jennifer, 2000, op. cit.

40. Ruíz GuRILlo, Leonor, 2012, op. cit.

41. HAY, Jennifer, 2000, op. cit.

42. KotTHOFF, Helga, 2006, op. cit.

43. Ruíz GuRILlo, Leonor, 2012, op. cit.

44. HOLMES, Janet. «Sharing a laugh: pragmatic aspects of humor and gender in the workplace». Journal of Pragmatics 38 (2006), pp. 26-50.

45. Kotthoff, Helga, 2006, op. cit.

46. Ruíz GuRILlo, Leonor, 2012, op. cit.

Feminismo/s 24, diciembre 2014, pp. 41-66 
los otros interlocutores y en muchas ocasiones sobre las mujeres ${ }^{47}{ }^{48}$. Las mujeres, sin embargo, raramente utilizan el humor para menospreciar al otro y marcar su propio estatus. De todas formas, el ataque al otro mediante el comentario humorístico también se da tanto en hombres como en mujeres, aunque siempre teniendo en cuenta la relación social y los factores contextuales. Las mujeres mayores muestran más permisividad hacia el humor agresivo sobre el otro, y las adolescentes usan el humor y la burla en sus conversaciones sobre $\operatorname{chicos}^{49} 50$.

Por último, las funciones psicológicas del humor también se podrían asociar a un rasgo tradicionalmente atribuido a las mujeres, puesto que su uso implica un estilo comunicativo más indirecto y probablemente más cortés, generalmente asociado a lo femenino ${ }^{51}$. Sin embargo, tanto hombres como mujeres utilizan el humor para minimizar el impacto de temas difíciles o manejar conflictos y contribuciones problemáticas en la interacción. Hay $(2000)^{52}$ encuentra que las mujeres recurren al humor de manera más general en todos los aspectos potencialmente problemáticos del discurso, mientras que los hombres lo hacen solo para aquellos casos surgidos durante la interacción.

\section{Relación social}

La segunda variable que vamos a considerar en este estudio es la relación social. El concepto de relación social no es nuevo en los estudios del discurso, sin embargo como indicábamos en la introducción, a pesar de constatarse su importancia en el uso de las expresiones lingüísticas, hasta el momento solo ha recibido una relativa atención.

Dentro de la sociolingüística se conoce desde hace tiempo la interacción entre los vínculos que unen a los hablantes y el uso de distintas expresiones

47. BENDIX, John y BENDIX, Regina. «Politics and gender in humor and satire: the cases of Elisabeth Kopp and Geraldine Ferraro.» Schweizer Zeitschrift für Soziologie, 2, (1992), pp. 441-460.

48. BARON, Bettina, y KotTHOFF, Helga (dirs.). Gender in Interaction: Perspectives on Femininity and Masculinity in Ethnography and Discourse, Amsterdam, John Benjamins, 2001.

49. EDER, Donna. "Go get ya a French!": romantic and sexual teasing among adolescent girls». En Deborah Tannen (dir), Gender and Conversational Interaction, Oxford, University Press, 1993, pp. 17-30.

50. KotTHOFF, Helga, 2006, op. cit.

51. Cameron, Deborah. The Myth of Mars and Venus: Do Men and Women Really Speak Different Languages? Oxford, University Press, 2007.

52. HAY, Jennifer, 2000, op. cit. 
lingüísticas. Por ejemplo, la conocida teoría de las redes sociales ${ }^{53}$ se sustenta en la idea de que el cambio lingüístico se produce en función de las relaciones interpersonales de los miembros de una comunidad. Otras corrientes que también han centrado su atención en la relación social se encuentran dentro de la Pragmática. La nueva corriente denominada Pragmática Interpersonal ${ }^{54}$ estudia 'la forma en la que los actantes usan el lenguaje para dar cuerpo y formar relaciones 'in situ' 55 . Tomando como base los postulados de la Comunicación Interpersonal, definen las relaciones interpersonales como «conexiones sociales mutuas entre individuos mediadas por la interacción, incluyendo el poder, la confianza, los roles, los derechos y las obligaciones $»^{56} 57$. Las relaciones pueden entenderse en su versión débil o fuerte ${ }^{58}$. La primera entiende la relación como la suma de las circunstancias de cada uno de los actantes, a partir de los objetivos y demandas de cada uno de ellos. La segunda la entiende como el resultado de la relación que une a los participantes y que se construye y modifica con cada una de las interacciones. La relación en este caso es dinámica y en continuo cambio y condiciona cada una de las interacciones futuras de esos individuos. La visión sin embargo más radical de la pragmática basada en las relaciones sociales es la defendida por Einfiel $(2009 ; 2013)^{59} 60$ en su teoría 'Relationship thinking' ${ }^{61}$. Desde una perspectiva cognitivo antropológica, Einfeld considera que el individuo se define prioritariamente en términos de sus relaciones sociales. Siguiendo las teorías clásicas de la comunicación, afirma que el ser humano al comunicarse debe sujetarse a dos imperativos pragmáticos básicos, el informacional y el afiliativo. El imperativo informacional persigue que aquello que se dice sea interpretado de acuerdo a nuestra intención comunicativa, mientras que el afiliativo nos hace asegurarnos de que manejamos adecuadamente las consecuencias socia-

53. Milroy, Lesley. Language and Social Networks, Blackwell, Oxford, 1987.

54. LOCHER, Miriam y GRAHAM, Sage L. «Introduction to interpersonal pragmatics». En Miriam Locher (dir.). Interpersonal Pragmatics. Berlin, Mouton de Gruyter, 2010, pp. $1-13$.

55. LOCHER, Miriam y GrahAm, Sage L., op. cit., p. 1.

56. HAUGH Michael, KÀDÀr Dániel Z. y Mills, Sara. «Interpersonal pragmatics. Issues and debates». Journal of Pragmatics 58 (2013), p. 4.

57. Traducción propia.

58. ARUNDALE, Robert, 2010, op. cit.

59. ENFIELD, Nick J. «Relationship thinking and human pragmatics». Journal of Pragmatics, 41 (2009), pp. 60-78.

60. ENFIELD, Nick J. Relationship thinking: Agency, enchrony, and human sociality. Oxford, University Press, 2013.

61. Hasta el momento no existe traducción para este término. Una aproximación sería «Pensamiento basado en las relaciones».

Feminismo/s 24, diciembre 2014, pp. 41-66 
les en nuestras interacciones ${ }^{62}$. Y es concretamente este último el que conduce al individuo a construir de forma particular su discurso, puesto que como seres sociales estamos obligados a mantener nuestras relaciones con los otros. Esto nos lleva a la configuración de una estructura social propia para la que poseemos restricciones cognitivas específicas; asimismo nos obliga a emplear tiempo y esfuerzo en pos de su mantenimiento.

Dentro de las corrientes hispánicas del discurso no existen trabajos que incorporen específicamente la variable «relación social», a excepción de algunas propuestas realizadas por Grupo Val.Es.Co. Briz (1998) ${ }^{63}$ en su caracterización del registro coloquial, propone contar con la llamada 'relación vivencial de proximidad' que engloba el «conocimiento mutuo, saber y experiencia compartidos (presuposiciones comunes) ${ }^{64}$. Como vemos, este rasgo podría considerarse cercano a la relación social entre los hablantes, puesto que alude a las características que estos comparten en función de su experiencia compartida, lo que tiene capacidad de condicionar la producción e interpretación del discurso. Estos autores, sin embargo, no desarrollan esta variable o especifican las condiciones para que se dé esta relación o las implicaciones de los distintos tipos de proximidad.

De cualquier modo, es evidente que existe una clara relación entre el tipo de vínculo que se crea entre los hablantes y las condiciones de la interacción, y esto tiene reflejo necesariamente en el uso de las expresiones lingüísticas. En este artículo defendemos la idea de que el ser humano es esencialmente un ser social que configura su propia identidad a partir de la pertenencia al grupo $^{65}$, las actividades y la imagen individuales existen por contraposición a las sociales ${ }^{66}{ }^{67}$. Por tanto las relaciones sociales se crean, existen y deben preservarse para la propia supervivencia. Entendemos la relación social en su versión 'fuerte', como el vínculo que se construye de manera dinámica entre los actantes a partir de cada interacción y de las experiencias compartidas. Al mismo tiempo vamos más allá en la estructura de las relaciones del indi-

62. ENFIELD, Nick J., 2009, op. cit., p. 72.

63. BRIZ GÓMEZ, Antonio. El español coloquial en la conversación: esbozo de pragmalingüística, Barcelona, Ariel, 1998.

64. BRIZ GÓMEZ, Antonio, 1998, op. cit, p. 41.

65. TAYLOR, Charles. Sources of the Self: The Making of the Modern Identity, Cambridge, University Press, 1989.

66. GofFMAn, Erving. Interaction Ritual. Essays on Face-to-face Behavior, Nueva York, Pantheon Books, 1967.

67. HeRITAGE, John y RAYMOND, Geoff. «The terms of agreement: indexing epistemic authority and subordination in talk-in-interaction». Social Psychology Quarterly 68 (2005), pp. 15-38. 
viduo y creemos que no solo se crean condiciones cognitivas específicas de cada relación ${ }^{68}$ para la producción e interpretación del discurso en interacción, sino que el tipo de vínculo conlleva la creación de normas lingüísticas y comportamentales, un código relacional propio para cada diada que permite gestionar adecuadamente la relación con el otro ${ }^{69}$.

La relación social se crea a partir de los elementos históricos que condicionan el vínculo entre los individuos, la forma de creación de la relación, su duración en el tiempo, y el grado de confianza o profundidad de la misma. La mayoría de las relaciones son establecidas de antemano, 'ancladas externamente ${ }^{70}$ tales como las relaciones familiares, vecinales o profesionales. En todos estos casos las reglas de interacción están preestablecidas de acuerdo al consenso de la comunidad en la que surgen. Sin embargo, la historia compartida es primordial para caracterizar cada relación. La extensión en el tiempo, el grado de confianza y la frecuencia e intensidad de las experiencias vividas en común determinan esa historia ${ }^{71}{ }^{72}$. El hecho de compartir experiencias afianza la relación y ayuda a crear un compromiso recíproco. Cuanto más cercana sea la relación, más esperable es que ambos interlocutores se comporten de manera recíproca en cuanto a derechos y obligaciones en la relación. Esto condiciona un comportamiento social y lingüístico, en el que determinadas actuaciones son esperables o admisibles en función del grado de compromiso con el otro.

Los factores relacionados con la jerarquización social de los hablantes condicionan igualmente la constitución de las reglas de intercambio. La distancia social configurada a través del estatus y el poder de los miembros ${ }^{73}$ modifican la manera de relacionarse de dos personas a partir de la posición que se marca para cada uno de ellos, dentro de su comunidad y dentro de la propia relación. Los aspectos situacionales (escenario, tema, propósito de la

68. ENFIELD, Nick J., 2009, op. cit.

69. Burleson, Brant, MeTTS, Sandra y KirCH, Michael W., 2000, op. cit.

70. ENFIELD, Nick J., 2009, op. cit.

71. FERRAND, Alexis. «Redes heterogéneas de discusión y pluralismo cognitivo». REDES. Revista hispana para el análisis de redes sociales. Vol.10, 2 (2006). http://revista-redes. rediris.es/pdf-vol10/vol10_2.pdf. Consultado el 19-03-2014.

72. BOURDON, Sylvain. «Relaciones sociales y trayectorias biográficas: hacia un enfoque comprensivo de los modos de influencia». REDES. Revista hispana para el análisis de redes sociales. 16, 6/2009. http://revista-redes.rediris.es/pdf-vol16/vol16_6.pdf. Consultado el 13-03-2014.

73. Brown, Penélope y Gillman, Albert. «Pronouns of power and solidarity». En Thomas Albert Sebeok (dir.), Style in Language. Cambridge, Mass.: MIT Press, 1960, pp. 253-276.

Feminismo/s 24, diciembre 2014, pp. 41-66 
interacción y características de los participantes, edad, sexo y nivel de instrucción) marcarán asimismo su comportamiento interaccional.

Finalmente, del mismo modo, la individualidad de los actantes tiene un gran peso. A pesar de compartir edad, sexo o nivel de instrucción, ninguno de nosotros actuamos de igual manera. Cada persona se comporta socialmente en sus propios términos. Esta individualidad se refleja en un modo personal de comportamiento condicionado por la personalidad y el temperamento: el estilo del individuo.

A medida que se produce el conocimiento mutuo y una historia común compartida, se irán estableciendo reglas de intercambio por las que se rijan los actantes. Las reglas de intercambio no son sin embargo estáticas, las interacciones diarias y el número de experiencias compartidas en distintos contextos, producen cambios que van determinando la naturaleza de la relación, y clasificando en cada momento determinados comportamientos como admisibles o inadmisibles «para esa relación».

Como señalamos en Romera y Campos Prats $(2014)^{74}$, a pesar de la aparente particularidad de estas reglas existe un rango de comportamientos determinados por los patrones culturales y las normas propias de la comunidad de práctica que marcan límites que los actantes no pueden sobrepasar (Fig. 1). Estos patrones actúan como marco bajo el que se establecen las reglas particulares de intercambio en una relación, más flexibles y variables.

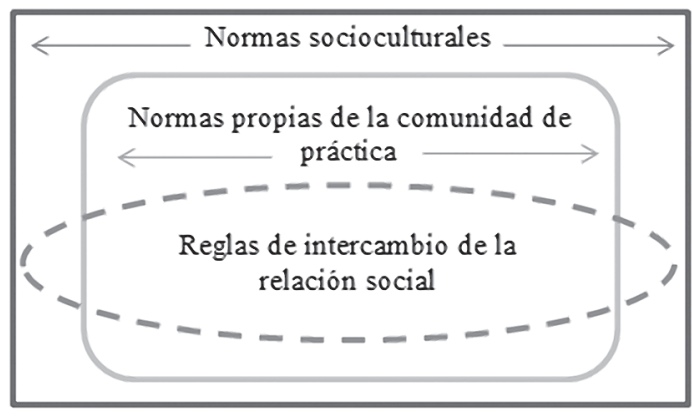

Figura 1. Reglas de intercambio

74. ROMERA, Magdalena y CAMPOS PRATS, Mar. Social relationships and discourse interaction: the case of disagreement in conversation. Enviado a Journal of Pragmatics. 2014. 
Como señala Einfield (2009) $)^{75}$, en toda relación participan además fuerzas negativas y positivas que marcan la interacción. En las relaciones no solo se promueve la confianza, o la identidad común, sino que también existe competencia, autoafirmación o diferenciación. Está claro que las características individuales de cada sujeto juegan aquí un papel importante. Debido al equilibrio de estas fuerzas, en cada interacción es habitual que se produzcan conflictos, especialmente amenazadores cuando afectan al futuro común de los relacionados. Cuando se produce una disonancia entre los individuos que crea tensión o conflicto entre ellos, los actantes pueden optar por la preservación de la relación o bien por asumir el riesgo del resquebrajamiento de la misma.

\section{Estudio empírico}

Los datos de este estudio están extraídos de conversaciones semidirigidas recopiladas entre hablantes de nivel de instrucción superior y edades comprendidas entre los 20 y los 55 años de la ciudad de Palma. Las grabaciones entre parejas se recogieron para la realización de un estudio que tenía como objetivo el análisis de las estrategias de cortesía lingüística en relación al género de los hablantes (Campos Prats, en preparación) ${ }^{76}$. El corpus cuenta con aproximadamente 7 horas de grabación y está compuesto por muestras de 14 hablantes adultos de distinto estatus, a los que se organizó e hizo interactuar por parejas dependiendo de los factores sociales género y tipo de relación. De estas, se tomaron 4 parejas. Además de estos datos, se recogieron grabaciones de otra pareja de amigos y de dos grupos de discusión mixtos, entre 18 y 25 años, todos ellos estudiantes o graduados universitarios. El primer grupo constaba de 3 mujeres y 1 hombre, y el segundo de 2 mujeres y 3 hombres. Tanto a las parejas como a los grupos se les pidió que discutieran sobre temas relacionados con los estereotipos de género.

La relación más o menos estrecha que mantenían los hablantes entre sí se determinó a partir de las siguientes variables:

1. Distancia social. Indica el grado de simetría que se establece en cuanto al estatus y el poder en la relación.

75. ENFIELD, Nick J., 2009, op. cit.

76. CAMPOS PRATS, Mar. Uso e impacto de las expresiones de des/acuerdo e identidad de género. Tesis doctoral. En preparación. 
2. Tipo de relación. Se tomaron tres tipos de relaciones de acuerdo a la división propuesta por Milardo (1988) ${ }^{77}$ y Milroy $(2008)^{78}$. Relación de intercambio en la que los hablantes mantienen una relación estrecha, interaccionan regularmente y además se prestan ayuda directa, consejo, crítica y apoyo. Relación transaccional en la que los hablantes interactúan quizá durante largos periodos de tiempo, pero no son fuente de apoyo material ni simbólico. Y relación pasiva, en la que los hablantes pueden no mantener interacción regularmente, pero sí constituyen ayuda y apoyo moral en los momentos en los que se necesita.

3. Grado de confianza. Tomado como el grado de intimidad y familiaridad entre los miembros de esa relación.

4. Calidad de la relación. Medida en función de lo bien o mal que se llevan los miembros (Alberts 1990) $)^{79}$.

5. Estilo de la pareja. Condicionado por las características de personalidad y temperamento de los miembros que resultará en la prevalencia de una de las dos fuerzas opuestas de confianza o diferenciación.

Todas las variables se ponderaron sobre el rango de 1 a 4 , donde 1 supone el valor de mayor distancia entre los interlocutores y 4 el valor de mayor cercanía entre los mismos. La ponderación dio lugar a la clasificación de hablantes y relaciones más o menos estrechas entre ellos, tal y como se muestra en la Tabla 1 a continuación.

Como vemos, las relaciones se sitúan en una escala de mayor o menor cercanía y fortaleza. Así las que se encuentran entre 1 y 2 son relaciones poco cercanas, entre 2 y 3 relativamente cercanas y entre 3 y 4 las más cercanas. En nuestros datos vemos que entre todos nuestros participantes hay relaciones que oscilan entre 2 y 4 , relativamente cercanas o muy cercanas, sin embargo se pueden establecer diferencias entre ellos. Entre cada pareja del mismo sexo una de ellas presenta mayor cercanía que la otra y lo mismo ocurre con los grupos, de modo que se puedan establecer diferencias con respecto a los resultados.

Las grabaciones fueron realizadas por un entrevistador o entrevistadora en cada caso, por lo que se trata de conversaciones semidirigidas en las que

77. MILARDO, Robert. «Families and social networks: an overview of theory and methodology». En Robert Milardo (dir.), Families and Social Networks, Newbury Park, California, Sage, 1988, pp. 13-47.

78. Milroy, Lesley. Social networks. En Jack. K. Chambers, Peter Trudgill y Natalie Schilling-Estes. The handbook of language variation and change. Oxford. Blackwell. 2008.

79. AlBERTS, Janet, 1990, op. cit. 
Tabla 1. Tipología de hablantes entrevistados y escala de relaciones entre los mismos

\begin{tabular}{|l|c|c|c|c|c|c|c|c|c|}
\hline Hablantes & Sexo & Edad & Vínculo & $\begin{array}{c}\text { Distancia } \\
\text { social }\end{array}$ & $\begin{array}{c}\text { Clase } \\
\text { de } \\
\text { relación }\end{array}$ & $\begin{array}{c}\text { Calidad } \\
\text { relación }\end{array}$ & $\begin{array}{c}\text { Grado de } \\
\text { confianza }\end{array}$ & $\begin{array}{c}\text { Estilo } \\
\text { pareja }\end{array}$ & $\begin{array}{c}\text { Relación } \\
\text { resultante }\end{array}$ \\
\hline Parejal & $\begin{array}{c}\text { Hombre } \\
\text { Mujer }\end{array}$ & $25-30$ & Pareja & 4 & 4 & 4 & 4 & 3 & 3,8 \\
\hline Pareja 2 & $\begin{array}{c}\text { Mujer } \\
\text { Mujer }\end{array}$ & $25-30$ & Amigas & 4 & 4 & 4 & 4 & 3 & 3,8 \\
\hline Pareja 3 & $\begin{array}{c}\text { Mujer } \\
\text { Mujer }\end{array}$ & $30-55$ & Madre-Hija & 3 & 4 & 4 & 3 & 3 & 3,4 \\
\hline Pareja 4 & $\begin{array}{l}\text { Hombre } \\
\text { Hombre }\end{array}$ & $18-25$ & Primos & 4 & 2 & 2 & 2 & 2 & 2,4 \\
\hline Pareja 5 & $\begin{array}{l}\text { Hombre } \\
\text { Hombre }\end{array}$ & $25-30$ & Amigos & 4 & 4 & 4 & 4 & 4 & 4 \\
\hline Grupo 1 & Mixto & $18-25$ & Compañeros & 4 & 2 & 2 & 2 & 2 & 2,4 \\
\hline Grupo 2 & Mixto & $25-30$ & Amigos & 4 & 4 & 3 & 3 & 3 & 3,4 \\
\hline
\end{tabular}

siempre se encontraba presente un miembro adicional al grupo o a la pareja. Sin embargo, es necesario señalar que en todos los casos este miembro pertenecía a la misma comunidad de habla que los participantes y mantenía con todos ellos una relación muy cercana que podría clasificarse del tipo 3-4 en nuestra escala de cercanía.

De las grabaciones se extrajeron todos aquellos fragmentos que contenían recursos al humor. Como criterio se tomó que hubieran producido una reacción de reconocimiento del humor entre los interlocutores, bien por risa o bien por reconocimiento explícito. Cada fragmento se codificó de acuerdo a las variables de los interlocutores, las variables contextuales, la estructura lingüística utilizada y sus funciones en el discurso. Las funciones se determinaron a partir de los tres tipos mencionados en la Sección 2, solidaridad, control y psicológico. Aunque ninguna de las funciones es excluyente y una misma expresión puede cumplir varias de ellas al mismo tiempo, se codificó la más prominente.

\section{5. resultados}

Lo primero que cabe destacar es que el recurso al humor presenta variaciones individuales importantes. Algunos sujetos recurren al mismo en numerosas ocasiones, es decir, forma parte de su modo personal de interacción, mientras que otros solo lo usan esporádicamente. Esto no se correlaciona con el 
género del individuo, en concreto 4 de nuestros participantes, 2 mujeres y 2 hombres, mostraron especial tendencia al modo humorístico de interacción, al menos en el contexto en el que se realizaron las grabaciones ${ }^{80}$. Esto nos ha ofrecido frecuencias de uso de expresiones humorísticas dispares, como se muestra en la Tabla 2.

Tabla 2. Frecuencia de uso de expresiones humorísticas en cada hablante

\begin{tabular}{|c|c|c|c|}
\hline Sexo & Pareja/Grupo & $\mathbf{n}$ & $\%$ \\
\hline H & P1 & 32 & 17,1 \\
\hline M & P2 & 27 & 14,4 \\
\hline H & P5 & 26 & 13,9 \\
\hline M & P2 & 17 & 9,1 \\
\hline H & P5 & 13 & 7,0 \\
\hline H & G1 & 13 & 7,0 \\
\hline M & P3 & 12 & 6,4 \\
\hline M & P3 & 11 & 5,9 \\
\hline H & P4 & 5 & 2,7 \\
\hline H & P4 & 5 & 2,7 \\
\hline M & G1 & 5 & 2,7 \\
\hline M & P1 & 4 & 2,1 \\
\hline H & G2 & 4 & 2,1 \\
\hline H & G2 & 4 & 2,1 \\
\hline M & G1 & 3 & 1,6 \\
\hline M & G1 & 3 & 1,6 \\
\hline M & G2 & 1 &, 5 \\
\hline M & G2 & 1 &, 5 \\
\hline H & G2 & 1 &, 5 \\
\hline & Total & 187 & 100,0 \\
\hline
\end{tabular}

El resto de los resultados confirman de igual manera la frecuencia de uso similar en mujeres y hombres; varían sin embargo las funciones con las que

80. Estamos de acuerdo con Kotthoff (2006), op. cit. en que la expresión del humor es extremadamente sensible al estado de ánimo y a las circunstancias concretas que atraviesa el individuo en cada momento. Por ello, consideramos que los resultados que ofrecemos pueden no ser extensibles a otros contextos. 
unas y otros las emplean. Las siguientes tablas 3 y 4 presentan los resultados obtenidos en los contextos de pareja y grupal.

Tabla 3. Funciones de la expresión de humor en mujeres y hombres en el contexto de parejas.

\begin{tabular}{|c|c|c|c|c|c|c|c|c|}
\hline & $\begin{array}{c}\text { Crear } \\
\text { consenso }\end{array}$ & $\begin{array}{c}\text { Expresar } \\
\text { desacuerdo }\end{array}$ & $\begin{array}{c}\text { Provocar } \\
\text { conflicto }\end{array}$ & $\begin{array}{c}\text { Manejar el } \\
\text { desacuerdo }\end{array}$ & $\begin{array}{c}\text { Crear } \\
\text { lazo } \\
\text { social }\end{array}$ & $\begin{array}{c}\text { Control } \\
\text { de las } \\
\text { normas }\end{array}$ & $\begin{array}{c}\text { Defensa/ } \\
\text { justificación }\end{array}$ & TOTAL \\
\hline MUJER & $32,4 \%$ & $4,2 \%$ & $2,8 \%$ & & $36,6 \%$ & $22,5 \%$ & $1,4 \%$ & $\begin{array}{c}100 \% \\
\mathrm{~N}=71\end{array}$ \\
\hline HOMBRE & $9,5 \%$ & $2,4 \%$ & $2,4 \%$ & $9,6 \%$ & $26,1 \%$ & $23,8 \%$ & $26,2 \%$ & $\begin{array}{c}100 \% \\
\mathrm{~N}=42\end{array}$ \\
\hline
\end{tabular}

Tabla 4. Funciones de la expresión de humor en mujeres y hombres en el contexto de grupo

\begin{tabular}{|c|c|c|c|c|c|c|c|c|}
\hline & $\begin{array}{c}\text { Crear } \\
\text { consenso }\end{array}$ & $\begin{array}{c}\text { Expresar } \\
\text { desacuerdo }\end{array}$ & $\begin{array}{c}\text { Provocar } \\
\text { conflicto }\end{array}$ & $\begin{array}{c}\text { Manejar el } \\
\text { desacuerdo }\end{array}$ & $\begin{array}{c}\text { Crear } \\
\text { lazo } \\
\text { social }\end{array}$ & $\begin{array}{c}\text { Control } \\
\text { de las } \\
\text { normas }\end{array}$ & $\begin{array}{c}\text { Defensa/ } \\
\text { justificación }\end{array}$ & TOTAL \\
\hline MUJER & $42,9 \%$ & $14,3 \%$ & & & $21,4 \%$ & $14,3 \%$ & $7,1 \%$ & $\begin{array}{c}100 \% \\
\mathrm{~N}=14\end{array}$ \\
\hline HOMBRE & $35,3 \%$ & $8,8 \%$ & $2,9 \%$ & $2,9 \%$ & $41,2 \%$ & $5,9 \%$ & $2,9 \%$ & $\begin{array}{c}100 \% \\
\mathrm{~N}=34\end{array}$ \\
\hline
\end{tabular}

Como vemos en la Tabla 3, en el contexto de pareja las mujeres utilizan el humor sobre todo en sus funciones sociales, para fortalecer el lazo social, y crear consenso, pero también para reforzar el control de las reglas de la interacción. Los hombres también refuerzan el vínculo social, pero prescinden del consenso y usan más el humor para el control y la justificación en aspectos problemáticos, como por ejemplo al cometer una equivocación en la conversación, como se muestra en el ejemplo (1).

$(1)^{81} \mathrm{~J}$ : Sí, aparte, creo que la mujer también lo hace, o sea creo que en esa situación estamos igual, tanto los chicos como las chicas salen de marcha.

81. En la transcripción de los ejemplos se han seguido las convenciones propuestas en DuBois et al. (1992). Estos son los símbolos básicos usados:

.. pausa breve

... pausa normal (cerca de un segundo)

... silencio

$=\quad$ alargamiento

@ risas

Feminismo/s 24, diciembre 2014, pp. 41-66 
P: [A ver, un momento, no, no lo hacen más, no lo hacen más. Lo hacen igual.

$\mathrm{J}: \quad$ [Yo he dicho igual]

P: Vale, a ver. Comunicación. Error (con voz robótica). Yo pensaba que no, yo pensaba que...

En el contexto grupal (Cf. Tabla 4 arriba), tanto hombres como mujeres utilizan el humor fundamentalmente para fortalecer los lazos sociales y crear consenso, aunque las mujeres también lo emplean para expresar el desacuerdo y mantener el control en la interacción. Parece entonces que a pesar de las diferencias, la función primordial del humor en todos los casos es reforzar los vínculos entre hablantes.

Al tener en cuenta la relación social que une a los hablantes observamos que los parámetros que más pesan son la cercanía de la relación y el poder en la pareja, como se muestra en la Tabla 5 a continuación.

Tabla 5. Funciones del humor entre las parejas de mujeres

\begin{tabular}{|c|c|c|c|c|c|c|c|}
\hline & & $\begin{array}{c}\text { Crear } \\
\text { consenso }\end{array}$ & $\begin{array}{c}\text { Crear } \\
\text { desacuerdo }\end{array}$ & $\begin{array}{l}\text { Provocar } \\
\text { conflicto }\end{array}$ & $\begin{array}{l}\text { Control de } \\
\text { las normas }\end{array}$ & $\begin{array}{c}\text { Crear lazo } \\
\text { social }\end{array}$ & $\mathbf{n}$ \\
\hline \multirow{2}{*}{ PAREJA 2} & AMIGAl & $\begin{array}{c}5 \\
29,4 \%\end{array}$ & & & & $\begin{array}{c}12 \\
70,6 \%\end{array}$ & 17 \\
\hline & AMIGA2 & $\begin{array}{c}11 \\
40,7 \%\end{array}$ & $\begin{array}{c}2 \\
7,4 \%\end{array}$ & & $\begin{array}{c}6 \\
22,2 \%\end{array}$ & $\begin{array}{c}8 \\
29,6 \%\end{array}$ & 27 \\
\hline \multirow{2}{*}{ PAREJA 3} & MADRE & $\begin{array}{c}2 \\
18,2 \%\end{array}$ & & $\begin{array}{c}2 \\
18,2 \%\end{array}$ & $\begin{array}{c}2 \\
18,2 \%\end{array}$ & $\begin{array}{c}5 \\
45,5 \%\end{array}$ & 11 \\
\hline & HIJA & $\begin{array}{c}5 \\
41,7 \%\end{array}$ & & & $\begin{array}{c}6 \\
50,0 \%\end{array}$ & $\begin{array}{c}1 \\
8,3 \%\end{array}$ & 12 \\
\hline
\end{tabular}

Como vemos, cuanto más cercana es la relación más expresiones de humor encontramos. Las dos parejas de mujeres obtienen resultados altos puesto que ambas mantienen relaciones muy cercanas. Ambas utilizan el humor de modo recurrente fundamentalmente para fortalecer su relación. Sin embargo, son las amigas las que más recurren a este modo de interacción. De las dos, A es quien lo utiliza más profusamente y presenta su uso en casi todas las funciones; A expresa acuerdo y desacuerdo, refuerza el lazo social y controla la interacción como se ve en (2), cuando interrumpe a M para introducir su intervención humorística:

negrita énfasis

[ ] segmento del discurso simultáneo con otro segmento dicho por otro hablante

/ truncamiento 
(2) M: (hablando de su novio) tiene= / tiene un interés súper extraño por la ropa y por la moda y por la elegancia; o sea, para él (.) venir elegante es/

A: $\quad$ iél no es italiano no?

En:@@@

M: No, no

A: $\quad$ entonces es gay

En:@@@

La pareja madre-hija también opta claramente por el fortalecimiento de la relación, aunque observamos que es la madre quien se permite el humor en la provocación del conflicto, debido a la asimetría existente, mientras que la hija lo usa para el refuerzo de su estatus mediante el control de las normas de la interacción. En (3) vemos como la madre se burla de la falta de delicadeza y atenciones del marido hacia la hija.

(3) M.: Bueno, un día] te regaló una piedra [@@@

En:_iiuna piedra?!] [@@@

V.: ¡Qué graciosa!]. [Era

M.: se fue] de excu[rsió=n@y le trajo una piedra@@

V.: $\quad \mathrm{No}==$ ], era una rosa del desierto que le habían traído [en un viaje.

M.: $\quad$ sí==!! ] ay, mira porque le había traído una piedra en vez de una rosa, de verdad!@@

V.: $\quad$ Es un hombre muy rudo, muy rural.

En:@@@

En el caso de los hombres las diferencias entre las dos parejas son más acusadas, en correlación con la distinta relación que los une (Cf. Tabla 6 a continuación).

Tabla 6. Funciones del humor entre las parejas de hombres

\begin{tabular}{|c|c|c|c|c|c|c|c|}
\hline & & $\begin{array}{c}\text { Crear } \\
\text { consenso }\end{array}$ & Desacuerdo & $\begin{array}{c}\text { Control } \\
\text { de las } \\
\text { normas }\end{array}$ & $\begin{array}{c}\text { Crear lazo } \\
\text { social }\end{array}$ & Justificación & $\mathbf{n}$ \\
\hline \multirow{2}{*}{ PAREJA 4} & PRIMOI & $\begin{array}{c}2 \\
40,0 \%\end{array}$ & & & & $\begin{array}{c}3 \\
60,0 \%\end{array}$ & 5 \\
\hline & PRIMO2 & $\begin{array}{c}1 \\
20,0 \%\end{array}$ & $\begin{array}{c}1 \\
20,0 \%\end{array}$ & & $\begin{array}{c}1 \\
20,0 \%\end{array}$ & $\begin{array}{c}2 \\
40,0 \%\end{array}$ & 5 \\
\hline \multirow{2}{*}{ PAREJA 5} & AMIGOl & & $\begin{array}{c}3 \\
23,1 \%\end{array}$ & $\begin{array}{c}4 \\
30,8 \%\end{array}$ & $\begin{array}{c}6 \\
46,2 \%\end{array}$ & & 13 \\
\hline & AMIGO2 & $\begin{array}{c}4 \\
30,8 \%\end{array}$ & $\begin{array}{c}1 \\
7,7 \%\end{array}$ & $\begin{array}{c}1 \\
7,7 \%\end{array}$ & $\begin{array}{c}6 \\
46,2 \%\end{array}$ & $\begin{array}{c}1 \\
7,7 \%\end{array}$ & 13 \\
\hline
\end{tabular}


Mientras que la pareja de amigos presenta el grado más alto de cercanía (4), los primos presentan el grado más bajo entre nuestras parejas $(2,4)$. Esta distancia es fundamental y explica los resultados en el uso del humor. La pareja 5 presenta un modo humorístico de relación en ambos miembros, y este modo de interacción es el que prevalece en todo momento. Se expresa el desacuerdo mediante el humor por ambos miembros, y prácticamente las expresiones humorísticas cumplen todas las funciones. Por el contrario la distancia entre los primos hace que las expresiones de humor sean más escasas y se usen en funciones de refuerzo social o de justificación, como se muestra en (4), donde C y D usan el humor ante una opinión que puede resultar difícil.

(4) C: (lee el tema del que se les pide que hablen)

«En todas las culturas las mujeres tienen menos interés en el atractivo visual del hombre que en el monetario»

C: Joer

D:@@@

C:1@@

D: $\quad$ Pfff. Está complicao este

Todos: @@@

Desde un punto de vista cualitativo, un aspecto interesante en el que la relación tiene un peso importante es en el uso del humor para la provocación del conflicto. Los casos que se han reseñado en otros estudios lo encuentran entre las parejas sentimentales (Alberts 1990). En nuestros datos, aparece efectivamente en la pareja hombre-mujer, pero también entre madre e hija. Las ocasiones en que esto ocurre no son muchas, 1 caso entre la pareja y 2 entre madre e hija. En este segundo caso, como se ha mostrado más arriba en (3), es la madre quien se burla de la hija en varias ocasiones a propósito de lo poco atento que es su yerno. El estatus asimétrico de la relación permite a la madre utilizar la burla con su hija, sabiendo no obstante que esto no pone en peligro la relación.

En caso de la pareja sentimental, es el hombre quien utiliza el humor para recriminar a la mujer su opinión acerca de ciertas mujeres. La recriminación provoca el conflicto conversacional durante numerosos turnos en los que ninguno de los dos da su brazo a torcer y es la entrevistadora quien finalmente zanja la discusión proponiendo una nueva pregunta.

$(5)^{82} \mathrm{~J}$ : A ver, siempre, en todas las sociedades se busca el ascenso social, una forma es casarte con un ricachón, digamos ino? @@ [...]

82. Debido a la longitud de la conversación se han mantenido los signos de transcripción más básicos y solo se ha reproducido la parte más pertinente del ejemplo. 
P: [Es que me estás contando una regla de, [@@] voy a hablar con tópicos ¿vale? porque = sí, porque me cuesta creer que en todas las sociedades lo que se lleva es ser «zorrón implacable». [@@]. O sea el zorrón implacable que se fija en el Ferrari antes que en el tal, cuando se dice que la mujer es emotiva y que busca la belleza en el interior. En el interior de la billetera. O sea que no.

Finalmente, la relación social se muestra relevante asimismo en la expresión del humor en el contexto grupal, como se ve en las Tablas 7 y 8 respectivamente.

Tabla 7. Funciones de la expresión de humor en mujeres y hombres en el grupo 1 con relación fuerte

\begin{tabular}{|c|c|c|c|c|c|c|c|c|}
\hline & $\begin{array}{c}\text { Crear } \\
\text { consenso }\end{array}$ & $\begin{array}{c}\text { Expresar } \\
\text { desacuerdo }\end{array}$ & $\begin{array}{c}\text { Provocar } \\
\text { conflicto }\end{array}$ & $\begin{array}{c}\text { Manejar el } \\
\text { desacuerdo }\end{array}$ & $\begin{array}{c}\text { Crear } \\
\text { lazo } \\
\text { social }\end{array}$ & $\begin{array}{c}\text { Control } \\
\text { de las } \\
\text { normas }\end{array}$ & $\begin{array}{c}\text { Defensa/ } \\
\text { justificación }\end{array}$ & TOTAL \\
\hline MUJER & $45,5 \%$ & $18,2 \%$ & & & $9,1 \%$ & $18,2 \%$ & $9,1 \%$ & $\begin{array}{c}100 \% \\
\mathrm{~N}=11\end{array}$ \\
\hline HOMBRE & $42,3 \%$ & $11,5 \%$ & $3,8 \%$ & $3,8 \%$ & $30,8 \%$ & $3,8 \%$ & $3,8 \%$ & $\begin{array}{c}100 \% \\
\mathrm{~N}=26\end{array}$ \\
\hline
\end{tabular}

Tabla 8. Funciones de la expresión de humor en mujeres y hombres en el grupo 2 con relación débil

\begin{tabular}{|c|c|c|c|c|c|c|c|c|}
\hline & $\begin{array}{c}\text { Crear } \\
\text { consenso }\end{array}$ & $\begin{array}{c}\text { Expresar } \\
\text { desacuerdo }\end{array}$ & $\begin{array}{c}\text { Provocar } \\
\text { conflicto }\end{array}$ & $\begin{array}{c}\text { Manejar el } \\
\text { desacuerdo }\end{array}$ & $\begin{array}{c}\text { Crear } \\
\text { lazo } \\
\text { social }\end{array}$ & $\begin{array}{c}\text { Control } \\
\text { de las } \\
\text { normas }\end{array}$ & $\begin{array}{c}\text { Defensa/ } \\
\text { justificación }\end{array}$ & TOTAL \\
\hline MUJER & $33,3 \%$ & & & & $66.7 \%$ & & & $\begin{array}{l}100 \% \\
\mathrm{~N}=3\end{array}$ \\
\hline HOMBRE & $12,5 \%$ & & & & $75 \%$ & $12,5 \%$ & & $\begin{array}{l}100 \% \\
\mathrm{~N}=8\end{array}$ \\
\hline
\end{tabular}

De nuevo, cuanto más cercana es la relación en el grupo, mayor número de expresiones de humor se usan y con un mayor rango de funciones. Como vemos, las diferencias entre ambos grupos son sustanciales. En el grupo con una relación fuerte, tanto mujeres como hombres refuerzan el vínculo con los otros a través del humor, pero se permite también la expresión del desacuerdo de modo humorístico y el control de las normas. Cuando la relación entre los hablantes del grupo es débil las funciones del humor son exclusivamente solidarias. 


\section{Conclusiones}

Parece, pues, que las expresiones humorísticas no están condicionadas por el género de los hablantes. Tanto hombres como mujeres emplean el modo humorístico de relación en sus interacciones y ambos las utilizan con frecuencia parecida. Lo que parece condicionar en mayor medida su uso es la relación social que mantienen los hablantes. Cuanto más cercana es la relación entre ellos, mayor es el recurso al humor en todas sus funciones. Nuestros datos muestran que la función primordial del humor es el refuerzo del lazo social que une a los hablantes, de modo que en relaciones menos cercanas se usa fundamentalmente para establecer y reforzar ese vínculo. Sin embargo, cuando la relación es estrecha, el abanico de funciones del humor se amplía y se emplea para la expresión del desacuerdo e incluso la provocación del conflicto tanto en hombres como en mujeres.

El contexto pareja/grupo actúa como regulador de las reglas de intercambio y diferenciador de las funciones con las que mujeres y hombres usan las expresiones humorísticas. En pareja, la mujer fundamentalmente crea consenso mediante el humor y refuerza la relación social, y cuanto más cercana es la relación más uso hace del humor para el resto de funciones, incluido el control de las reglas de la interacción y la expresión del desacuerdo. Asimismo en relaciones muy cercanas, la mujer puede provocar el conflicto pero midiendo el impacto de los actos lingüísticos, sin transgresión de los límites de la relación y siempre a sabiendas de que se llegará al consenso. El hombre también utiliza el humor como refuerzo del lazo social y expresión del desacuerdo en relaciones estrechas, pero también usa el humor como medio de autojustificación ante la expresión de aspectos problemáticos en relaciones menos cercanas. Esta última función, que en principio podría parecer perteneciente al estilo más indirecto, relacionado generalmente con el estilo femenino, en nuestros datos solo aparece usada por los hombres. En grupo pesa de nuevo la fuerza de la relación. En el grupo con relación estrecha, las mujeres crean consenso, expresan desacuerdo y realizan el control de las normas de interacción, mientras que los hombres expresan el consenso y refuerzan los lazos sociales. Si la relación es débil, tanto mujeres como hombres usan el humor únicamente en su función primaria de refuerzo de la relación.

\section{Referencias bibliográficas}

AlBERTS, Janet. «The use of humor in managing couples' conflict interactions».

En Cahn, Dudley D. (dir.). Intimates in conflict. A communicative prespective, Hillsdade, Lawrence Erlbaum, 1990, pp. 105-120. 
Alvarado Ortega, M. Belén. «An Approach to Verbal Humor in Interaction», Procedia-Social and Behavioral Sciences 95 C, (2013), pp. 594-603.

ARUndale, Robert. «Relating», En Miriam Locher y George Sage (dirs.): Interpersonal pragmatics, Berlin, Mouton de Gruyter, (2010), pp. 137-165

AtTARDo, Salvatore. Humorous Texts: A Semantic and Pragmatic Analysis, Nueva York, Mouton de Gruyter, 2001.

ATtARDO, Salvatore. "A primer for the linguistics of humor.» En Victor Raskin (dir.). The primer of humor research, Berlin, Mouton de Gruyter, 2008.

BARON, Bettina, y KotTHOFF, Helga (dirs.). Gender in Interaction: Perspectives on Femininity and Masculinity in Ethnography and Discourse, Amsterdam, John Benjamins, 2001.

BENDIX, John y BENDIX, Regina. «Politics and gender in humor and satire: the cases of Elisabeth Kopp and Geraldine Ferraro.» Schweizer Zeitschrift für Soziologie, 2, (1992), pp. 441-460.

BOURDON, Sylvain. «Relaciones sociales y trayectorias biográficas: hacia un enfoque comprensivo de los modos de influencia». REDES. Revista hispana para el análisis de redes sociales. 16, 6/2009. http://revista-redes.rediris.es/pdf-vol16/ vol16_6.pdf consultado el 13-03-2014.

BRIZ GÓMEZ, Antonio. El español coloquial en la conversación: esbozo de pragmalingüística, Barcelona, Ariel, 1998.

Brown, Penélope y GiLlman, Albert. «Pronouns of power and solidarity». En Thomas Albert Sebeok (dir.), Style in Language. Cambridge, Mass.: MIT Press, 1960, pp. 253-276

Burleson, Brant, MetTs, Sandra y KirCH, Michael W. "Communication in close relationships». En Clyde Hendrick y Susan S. Hendrick (dirs.), Close relationships: A sourcebook, Thousand Oaks, CA, Sage, 2000, pp. 244-258.

CAmeron, Deborah. The Myth of Mars and Venus: Do Men and Women Really Speak Different Languages? Oxford, University Press, 2007.

CAMPos PRATS, Mar. Uso e impacto de las expresiones de des/acuerdo e identidad de género. Tesis doctoral. En preparación.

COSER, Rose L. «Laughter among colleagues». Psychiatry 23 (1960), pp. 81-95.

Cox, Joe, A. ReAd, L. Raymond y VAn Auken, Philip M., «Male-female differences in communicating job-related humor: An exploratory study». Humor 3(1990), pp. 287-295.

DEAUX, Kay, «Sex and Gender». Annual Review of Psychology 36 (1995), pp. 49-82.

DEGENNE Alain y FORSE, Michel. Les réseaux sociaux, une analyse structurale en sociologie, París, Armand Colin, 2004.

Dubois, John, Schuetze-Coburn, Stephan, Cumming, Susanne y Paolino, Danae: «Outline of Discourse Transcription». En Jane Edwards y Martin Lampert, 
(dirs.): Talking Data. Transcription and Coding in Discourse Research. Hillsdale, Erlbaum, 1992, 45-89.

EDER, Donna. «"Go get ya a French!”: romantic and sexual teasing among adolescent girls». En Deborah Tannen (dir), Gender and Conversational Interaction, Oxford, University Press, 1993, pp. 17-30.

ENFIELD, Nick J. «Relationship thinking and human pragmatics». Journal of Pragmatics, 41 (2009), pp. 60-78.

ENFIELD, Nick J. Relationship thinking: Agency, enchrony, and human sociality. Oxford, University Press, 2013.

ERVIN-TripP, Susan M., LAMPERT, Martin D. «Gender differences in the construction of humorous talk». En Kira Hall, Mary Buchholtz y Birch Moonwomon (dirs.), Locating Power: Proceedings of the Second Berkeley Women and Language Conference. Berkeley, University of California, 1992, pp. 108-117.

FERRAND, Alexis. «Redes heterogéneas de discusión y pluralismo cognitivo». REDES. Revista hispana para el análisis de redes sociales. Vol.10, 2 (2006). http:// revista-redes.rediris.es/pdf-vol10/vol10_2.pdf consultado el 19-03-2014.

FINCH, Janet y MASON, Jennifer. Negotiating Family Responsibilities, Londres, Routledge, 1993.

GofFMAn, Erving. Interaction Ritual. Essays on Face-to-face Behavior, Nueva York, Pantheon Books, 1967.

GOODMAN, Lizbeth. «Gender and humour». En Frances Bonner et al. (dirs), Imagining women: Cultural representations and gender, Cambridge: Polity, 1992, pp. 296-300.

Grice, H. Paul. «Logic and conversation». En Peter Cole y Jerry Morgan (dirs.), Syntax and Semantics 3: Speech acts, Nueva York, Academic Press, 1975, pp. 41-58.

GrossetTI, Michel. «Where do social relations come from?: A study of personal networks in the Toulouse area of France». Social Networks 27 (2005), pp. 289-300.

HAUGH Michael, KÀDÀr Dániel Z. y MiLlS, Sara. «Interpersonal pragmatics. Issues and debates». Journal of Pragmatics 58 (2013), pp. 1-11.

HAY, Jennifer. «Functions of humor in the conversations of men and women». Journal of Pragmatics 32 (2000), pp. 709-742.

HERITAGE, John y RAYMOND, Geoff. «The terms of agreement: indexing epistemic authority and subordination in talk-in-interaction». Social Psychology Quarterly 68 (2005), pp. 15-38.

HOLMES, Janet. «Sharing a laugh: pragmatic aspects of humor and gender in the workplace». Journal of Pragmatics 38 (2006), pp. 26-50.

JENKINS, Mercilee. «What's so funny?: Joking among women». En Sue Bremner, Noelle Caskey y Birch Moonwomon (dirs), Proceedings of the first Berkeley 
Women and Language Conference, Berkeley, CA, Women and Language Group, 1985, pp. 135-151.

Kerbrat-Orecchioni, Catherine. «L'humour au quotidien». En Philip Merlo (dir.) L'humour hispanique, Grimh-LCE-Grimia, Université Lumière Lyon 2, 2004.

KotThoff, Helga. «Gender and joking. On the complexities of women's image politics in humorous narratives». Journal of Pragmatics 32 (2000), pp. 55-80.

KotTKOFF, Helga. «Gender and humor: The state of the art». Journal of Pragmatics 38 (2006), pp. 4-25.

LAKOFF, Robin. Language and Woman's Place. NuevaYork, Harper y Row, 1975.

LOCHER, Miriam y GRAHAM, Sage L. «Introduction to interpersonal pragmatics». En Miriam Locher (dir.). Interpersonal Pragmatics. Berlin, Mouton de Gruyter, 2010, pp. 1-13.

MARTIN, Rod A. The psychology of humor. An integrated approach, Amsterdam, Elsevier, 2007.

MARTINEAU, William. «A model of the social functions of humor». En Jeffrey Goldstein y Paul McGhee (dirs.), The psychology of humor,. Nueva York, Academic Press, 1972, pp. 101-125.

MILARDO, Robert. «Families and social networks: an overview of theory and methodology». En Robert Milardo (dir.), Families and Social Networks, Newbury Park, California, Sage, 1988, pp. 13-47.

Milroy, Lesley. Language and Social Networks, Blackwell, Oxford, 1987.

Milroy, Lesley. Social networks. En Jack. K. Chambers, Peter Trudgill y Natalie Schilling-Estes. The handbook of language variation and change. Oxford. Blackwell. 2008.

MitCHell, J. Clyde. «Network procedures». En Dieter Frick (dir.), The Quality of Urban Life, Berlin, Mouton de Gruyter, 1986, pp. 73-92.

Mulkay, Michael. «On Humour», Cambridge, Polity Press, 1988.

PULLIN, Patricia. «Small talk, rapport, and international communicative competence: Lessons to learn from BELF». Journal of Business Communication, 47 (4) (2010), pp. 455-476.

RASKIN,Victor. Semantic Mechanisms of Humor. Dordrecht, D. Reidel, 1985.

ROMERA, Magdalena y CAMPOS PRATS, Mar Social relationships and discourse interaction: the case of disagreement in conversation. Enviado a Journal of Pragmatics. 2014.

Ruíz GuRILlo, Leonor. La lingüística del humor en español, Madrid, Arco/Libros, 2012.

Ruíz Gurillo, Leonor y Padilla García, Xosé. A. (dirs.). Dime cómo ironizas y te diré quién eres. Una aproximación pragmática a la ironía, Frankfurt, Peter Lang, 2009. 
TAYLOR, Charles. Sources of the Self: The Making of the Modern Identity, Cambridge, University Press, 1989.

Watzlawick, Paul, Beavin, Janet y Jackson, Don. The Pragmatics of Human Communication. Nueva York, W.W. Norton and Company, 1967. 